Open Access to Pharmaceutical and Medical Research

(C) 2011-18, publisher and licensee JDDT, This is an Open Access article which permits unrestricted non-commercial use, provided the original work is properly cited

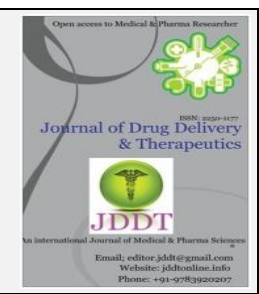

Open $\odot$ Access

Review Article

\title{
A Review on Mouth Dissolving Film
}

\section{Pawar Rajat*, Sharma Ravi, Sharma Pravin, Darwhekar G.N.}

Acropolis Institute of pharmaceutical Education and Research, Indore, M.P., India

\begin{abstract}
Mouth dissolving film is the most advanced oral solid dosage form due to its flexibility and comfort in use. Mouth dissolving films are oral solid dosage form that disintegrate and dissolve within a minute when placed in mouth without taking water or chewing. This dosage form allows the medication to bypass the first pass metabolism so bioavailability of medication may be improved .Mouth dissolving film has potential to improve onset of action lower the dosing and eliminate the fear of chocking. Formulation of mouth dissolving films involves both the visual and performance characteristics as plasticized hydrocolloids, API taste masking agents are being laminated by solvent casting and semisolid casting method. Solvent casting method being the most preferred method over other methods because it offers great uniformity of thickness and films prepared having fine glossy look and better physical properties. Mouth dissolving films are evaluated for its various parameters like thickness, physical property like folding endurance, disintegration and dissolution time. This review gives an idea about formulation techniques, evaluation parameters, overview on packaging and some available marketed products of mouth dissolving films.
\end{abstract}

Keywords: Mouth dissolving film, solvent casting, fast disintegration

Article Info: Received 05 Sep 2019; Review Completed 18 Oct 2019; Accepted 26 Oct 2019; Available online 15 Nov 2019

\section{Cite this article as:}

Pawar R, Sharma R, Sharma P, Darwhekar GN, A Review on Mouth Dissolving Film, Journal of Drug Delivery and Therapeutics. 2019; 9(6):206-210 http://dx.doi.org/10.22270/jddt.v9i6.3676

\section{*Address for Correspondence:}

Pawar Rajat, Acropolis Institute of pharmaceutical Education and Research, Indore, M.P., India

\section{INTRODUCTION:}

Oral route of drug administration is a most preferred route due to its ease of administration, non-invasiveness, adaptability, patient compliance and acceptability. Regarding oral route of drug administration, many substitutes have continuously been presented by using recent novel technologies for pediatrics, geriatrics, nauseous and non-compliance patients. Bioadhesive mucosal dosage forms including adhesive tablets, gels and patches are outcomes of technological development. Among various dosage forms, the use of polymeric films for delivering medication into buccal cavity has developed great potential in recent area. Orally disintegrating films (ODFs), when placed on tongue, immediately hydrates by soaking saliva following disintegration and/or dissolution releasing active pharmaceutical agent from the dosage form. ODFs are kind of formulations which are commonly prepared using hydrophilic polymers enabling rapid dissolution upon contact with saliva. Oral disintegrating tablets (ODTs) and oral disintegrating films (ODFs) are the typical examples of orally disintegrating drug delivery systems. These systems were developed in late 1970 to serve as an alternative to conventional dosage forms, for instance, fast disintegrating tablets and capsules for geriatrics and pediatric patients having difficulty in swallowing conventional dosage forms. A typical ODF is usually equal to the size of a postage stamp[1]. In market place, the introduction of ODT was strongly associated with counseling of patients about the appropriate administration by giving instruction like "do not chew/do not swallow". However, in spite of these instructions, incidents regarding chewing and swallowing were often reported. But, ODFs untied the masses from these adverse events. The administration of ODFs has numerous advantages and some of them are as follows:

\section{i. Easy transportation.}

ii. Ease of swallowing for geriatrics and pediatrics.

iii. Convenient and accurate dosing.

iv. No need of water for administration.

v. Convenient for dysphasic patients having difficulty in swallowing tablets and capsules.

vi. Rapid onset of action with increased bioavailability due to bypassing hepatic first pass effect and stability

No expensive lyophilization, high mechanical strength, rapid disintegration, and reduced choking risks are the quality attributes of ODFs21a have attained remarkable significance in pharmaceutical industry for the reason of possessing unique properties and fast disintegration time ranging from seconds to one minute ODFs design permits to incorporate a variety of drugs for their pharmacological effects e.g., antitussive, anti-epileptic, anti-asthmatic, expectorant, etc. .High temperature and moisture [2]. 


\section{SPECIAL FEATURES OF MOUTH DISSOLVING FILMS}

1. Thin elegant film

2. Unconstructive

3. Available in various size and shapes

4. Fast disintegration

5. Rapid release

6. Give a pleasant mouth feel.

7. Have an acceptable taste.

8. Should not leave residues in mouth.

\section{DISADVANTAGES}

1. Dose uniformity is a technical challenge.

2. Hygroscopic in nature.

3. High doses cannot be incorporated $(<40 \mathrm{mg} / 4 \mathrm{~cm} 2$ piece)

4. Require special packaging for products stability and safety.

\section{COMPOSITION OF MOUTH DISSOLVING FILM [3]:}

\begin{tabular}{|l|l|}
\hline \multicolumn{1}{|c|}{ Composition } & Concentration \\
\hline Drug & $1-25 \%$ \\
\hline Water soluble polymer & $40-50 \%$ \\
\hline Plasticizers & $0-20 \%$ \\
\hline Fillers, colors ,flavors & $0-40 \%$ \\
\hline
\end{tabular}

\section{FORMULATION ASPECTS FOR MOUTH DISSOLVING FILMS:}

\section{Active Pharmaceutical Ingredient:}

Various classes of drugs can be incorporated into ODFs e.g., anti-histamine, anti-diarrheal, anti-depressants, vasodilators, anti-asthmatic, anti-emetic, etc. Dimenhydrinate can also be incorporated into ODFs for taste masking. Common examples of drugs incorporated into ODFs are salbutamol sulfate, rizatriptan benzoate, verapamil ondansetron, dexamethasone, rofecoxib, cetirizine, pilocarpine, tianeptine sodium, indomethacin, etc.

\section{Film Forming Polymer:}

Water-soluble polymers are used as film formers as they provide quick disintegration, good mouth feel, and mechanical strength to the films. The robustness of the strip depends on the type of polymer and its amount in the formulations. A variety of polymers are available for preparation of films of which pullulan, gelatin and hypromellose are most commonly used. Examples of watersoluble polymers include: Pullulan, Gelatin, guar gum, xanthan gum, Hydroxyl propyl methyl cellulose (HPMC), Modified starches, PVPK30, PVA etc. HPMC E3/E5/E6/E15.

Ideal properties of the polymers used in the oral film:

1. Polymers should be nontoxic, non- irritant and non-bitter.

2. Polymers should be tasteless

3. It should be devoid of leachable impurities

4. It should be inexpensive and readily available
5. It should not be an obstacle in the disintegration time 6. It should have good wetting and spreadibility property

7. It should exhibit sufficient peel, shear and tensile strength

8. It should not cause secondary infection in the oral cavity and should have sufficient shelf life.[4]

\section{Plastisizers:}

In general, mechanical properties such as tensile strength and percent elongation are improved by adding plasticizer to the formulations. The concentration of plasticizer usually ranges from $0 \%$ to $20 \% \mathrm{w} / \mathrm{w}$. Common examples of plasticizers are PEG, glycerol, diethyl phthalate, triethyl citrate, tributyl citrate, etc.

\section{Sweetening Agent:}

Sweeteners have become an important part of the food products as well as pharmaceutical products intended to be disintegrated or dissolved in the oral cavity. Natural sweeteners as well as artificial sweeteners are used to improve the palatability of the mouth dissolving formulations. Some suitable sweeteners include:

(1) Water soluble natural sweetener: xylose, ribose, glucose, sucrose, maltose, stevioside etc.

(2) Water soluble artificial sweetener: sodium or calcium saccharin salts, acesulfame-K etc.

(3) Dipeptide based sweetener: aspartame

\section{Saliva Stimulating Agent:}

Salivary stimulants are generally acidic in nature stimulating the production of saliva in buccal cavity, consequently, promoting the disintegrating of ODFs. Some commonly used saliva stimulating agents are citric acid, malic acid, tartaric acid, ascorbic acid and lactic acid.

Surfactant: Surfactants are used as solubilizing or wetting or dispersing agents as a result that the film gets dissolved within seconds and release active agent immediately. Surfactants also improve the solubility of poorly soluble drugs in fast dissolving buccal films. E.g.: Polaxamer 407, sodium lauryl sulfate, benzalkonium chloride, benzthonium chloride, tweens and spans etc.

Flavor: Flavors are needed to mask the bitter or nauseating taste of incorporated drug. Amount of flavor depends upon its nature and strength. Any US-FDA approved flavor can be used such as sweet, sour or mint flavor one of the research work verified that mint, licorice and sucralose mixture flavors appropriately mask the bitter taste of diclofenac sodium. Electronic tongues are used to discriminate the effect of various taste masking agents (TMAs)

Colouring Agent: Pigments such as titanium dioxide or FD\&C approved coloring agents are incorporated (not exceeding concentration levels of $1 \% \mathrm{w} / \mathrm{w}$ ) in oral strips when some of the formulation ingredients or drugs are present in insoluble or suspension form[5].

\section{METHOD OF PREPARATION OF FAST DISSOLVING FILM:}

One or a combination of the following processes can be used to manufacture the Mouth dissolving film:

1. Solvent casting

2. Hot-melt extrusion

3. Semisolid casting 


\section{Solid dispersion extrusion}

\section{Rolling}

\section{Solvent Casting Method:}

Solvent casting is the most commonly used method for the preparation of ODFs using water soluble excipients, polymers and drug which are dissolved in de-ionized water; consequently, a homogenous mixture is obtained by applying high shear forces generated by a shear processor. Then, the prepared solution is poured onto petri plate and the solvent is allowed to dry by exposing it to high temperature in order to attain good quality films. An orodispersible film of tianeptine sodium was successfully prepared through solvent casting technique using different grades of Lycoat and HPMC. In solvent casting technique, film forming polymer is usually soaked in an appropriate solvent for overnight. The type of API, which has to be incorporated in ODF, governs the selection of a suitable solvent depending on critical physico- chemical properties of API such as melting point, shear sensitivity and polymorphic form. Compatibility of drug with solvent and other excipients is also brought under consideration before finalizing a formulation. During formulation, entrapment of air bubbles can hinder the uniformity of prepared films. Thus, deaeration of the mixture is carried out with the help of a vacuum pump. Orodispersible film formulation of mosapride was also successfully prepared by using solvent casting method. Viscosity of the solution to be poured is an imperative aspect in casting method. The concentration of pullulan varying from $2 \%$ to $8 \%$ results into low viscosity solution, as a result, enabling easy casting of films. Fast disintegrating films of anastrozole were also effectively prepared with the help of solvent casting method employing HPMC (E5) and polyvinyl alcohol (PVA) ${ }^{[6,7] .}$

\section{Hot Melt Extrusion:}

Hot melt extrusion is a technique in which a mixture containing drug, polymer and excipients is extruded under high temperature to form a homogenous mass which is then coasted to form smooth films. This is a solvent free process; however, the processing of thermolabile substances is a major drawback of this process due to the use of high temperature during extrusion
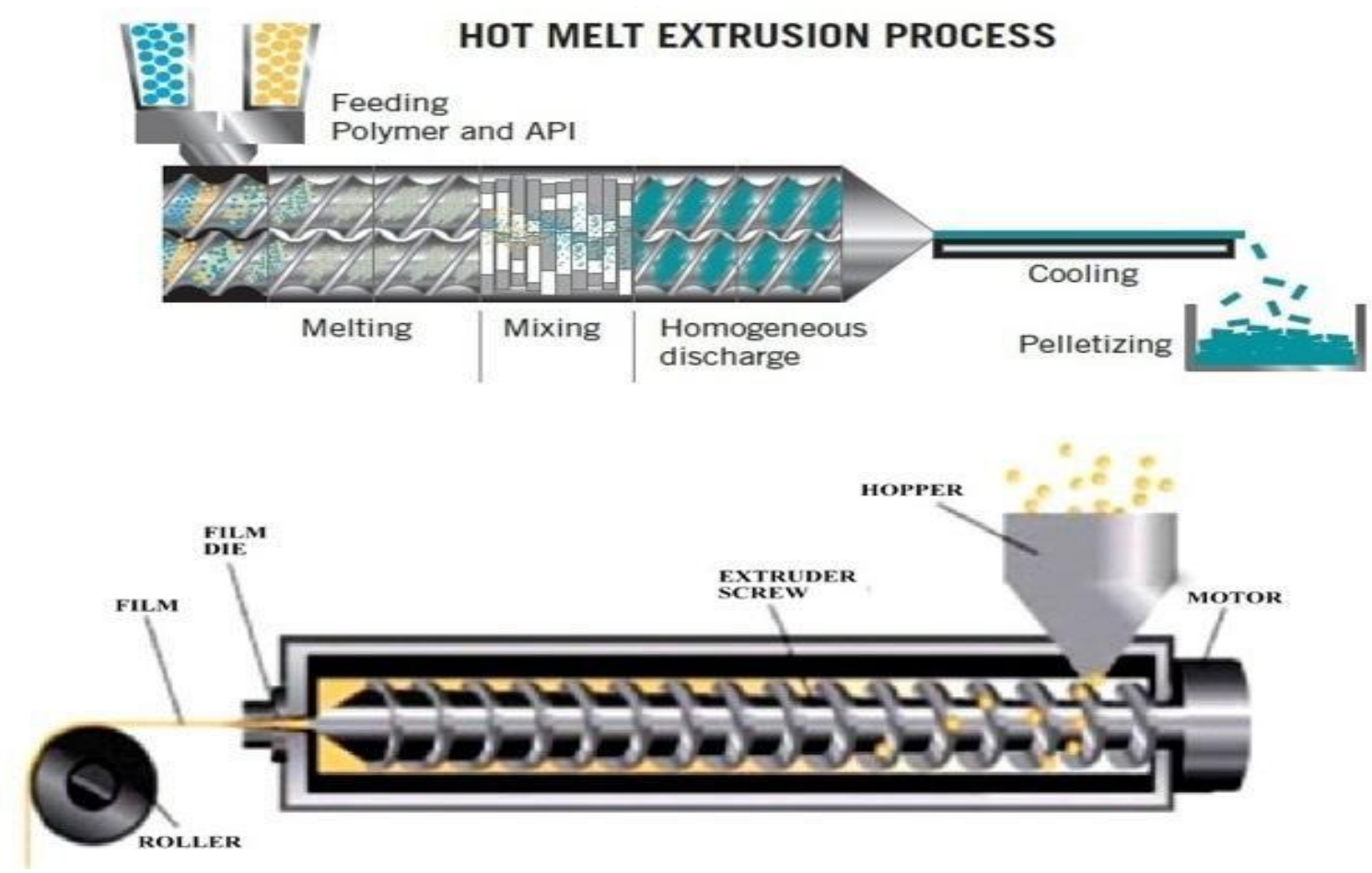

\section{Semi Solid Casting Method:}

This method is preferably adopted when acid insoluble polymers are to be used in the preparation of the films. Acid insoluble polymers used to prepare films include: cellulose acetate phthalate, cellulose acetate butyrate. Acid insoluble polymer and film forming polymer should be used in the ratio of 1:4.

Solution of water soluble film forming polymer is prepared

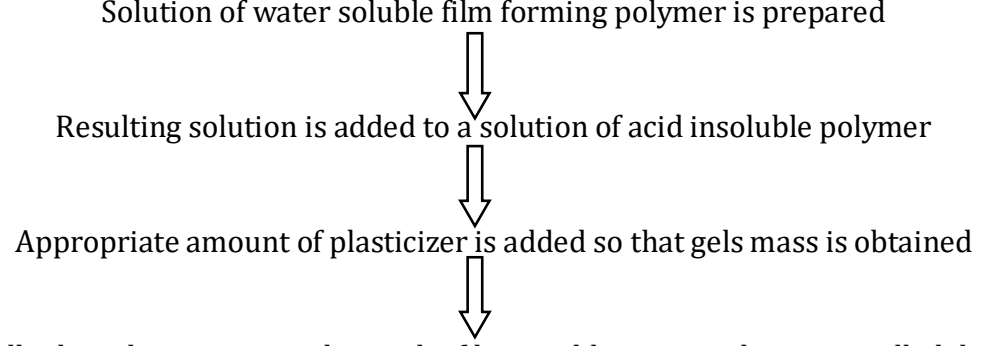

Finally the gel mass is casted in to the film or ribbons using heat controlled drums 


\section{Solid Dispersion Extrusion Method:}

Solid dispersion of domperidone using beta-cyclodextrin, PEG400 and HPMC E15 was successfully prepared and films were casted using solid dispersion extrusion method. $[9,10]$

\section{Solid dispersions}
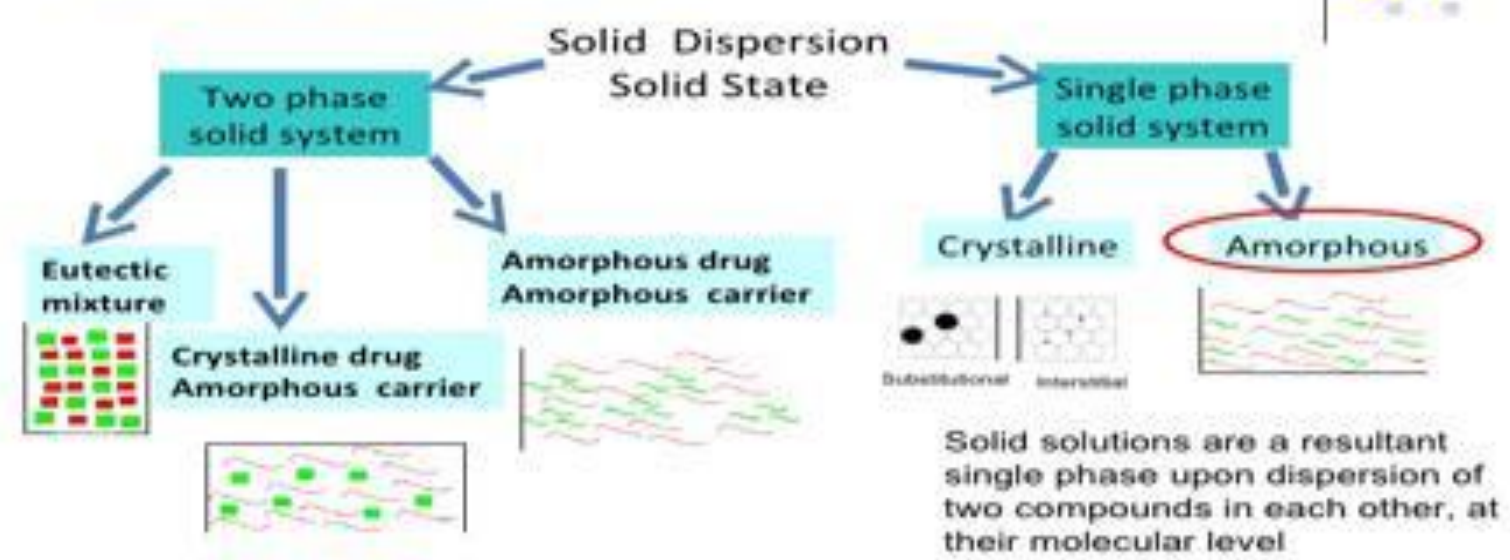

\section{Rolling Method [11, 12]:}

Plot of rolling method is prepared solution should possess specific rheological properties for rolling onto the drum.

Preparation of suspension of drug and polymer in water or alcohol Suspension is subjected to rollers Suspension is subjected to rollers Evaporation of solvent Evaporation of solvent

\section{EVALUATION PARAMETERS:}

Thickness test: Thickness of a film is determined by using calibrated digital micrometer and then subsequently mean average is calculated. Generally, three readings from all the batches are determined and average is calculated. Weight variation of a film is calculated in triplicate by cutting the film and determining weight of each film. Uniformity in thickness is important to as certain as it is directly proportional to dose accuracy of the film[13].

Tack test: Tack is the tenacity with which the film adheres to the accessory that has been pressed into contact with strip. This test also determines the dryness[14].

Tensile strength: Tensile strength is defined as maximum stress applied at which the film breaks. Basically, this test is performed to measure the mechanical strength of films. It can be calculated from applied load at rupture divided by the strip cross-sectional area given in the equation below[14,15]:

Tensile strength $=$ Load at breakage $/$ Strip thickness $\times$ Strip Width

Percentage elongation: When the sample films are subjected to tensile stress, deformation of the films occurs resulting in stretching or elongation of sample. It is performed to predict the ductility of polymers using a texture analyzer. It is calculated by formula:

$\%$ Elongation $=$ Increase in length $\times 100 /$ Original length

Folding endurance: To determine folding endurance, a portion of film is cut and repeatedly folded at the same point till it breaks. The number of times the film could be folded at the same point without breaking indicates the folding endurance value. Typical folding endurance for a film ranges between 100-150[16]

Swelling property: Simulated saliva solution is used to check the swelling studies of films. Initial weight of film is determined and is placed in pre weighed stainless steel wire mesh. This mesh containing film is then dipped into simulated saliva solution. Increase in the weight of film is noted at constant pre-determined time intervals until no more increase in weight. Degree of swelling is determined by these parameters:

Degree of swelling = final weight $(w t)-$ Initial weight $(w 0) /$ Initial weight (w0)

$\mathrm{Wt}=$ weight of film at time interval $\mathrm{t}, \mathrm{w} 0=$ weight of film at time 0 .

Surface pH: The $\mathrm{pH}$ value of a film is usually determined by putting the prepared film in Petri dish and subsequently film is made wet by using distilled water and noting $\mathrm{pH}$ by touching the film surface with a $\mathrm{pH}$ meter electrode. Determination of surface $\mathrm{pH}$ is vital as acidic or basic $\mathrm{pH}$ is liable to cause oral mucosal irritation.[16,17]

Content uniformity: Contents of a film are determined by standard assay method specified for individual drug in different pharmacopoeia. This test is performed on 20 samples using analytical techniques. The acceptance value of the test is less than $15 \%$ in accordance with Japanese pharmacopoeia. According to USP27, the contents should range from $85 \%$ to $115 \%$ with the standard deviation of less than or equal to $6 \%$ Content uniformity is worked out for estimating drug contents in individual film[18, 19].

Disintegration time: Disintegration apparatus mentioned in official pharmacopoeias is used for determining the disintegration time of a film. Normally, the disintegration time is the function of composition of film as it varies with the formulation and generally ranges from 5 to $30 \mathrm{~s}$. Mostly, the USP disintegration apparatus is used for this test. There are no official guidelines available for determining disintegration time of orally fast disintegrating films. There 
are two methods for determining disintegration time of film[20].

Slide frame method: A drop of distilled water is poured onto the film clamped into slide frames placed on petri dish. Time taken by the film to dissolves noted[21].

Petri dish method: A film is placed into $2 \mathrm{ml}$ distilled water taken in Petri dish. Time taken by the film to dissolve completely is considered as the disintegrating [22].

In-vitro dissolution test: Standard official basket or paddle apparatus is used for conducting dissolution studies on films. Sink conditions should be maintained during dissolution. Sometimes while performing this process, film floats over the medium making it difficult to perform the test properly. This problem is more likely to occur in case of paddle method thus the basket apparatus is mostly preferred. Media used are $6.8 \mathrm{pH}$ phosphate buffer $(300 \mathrm{ml})$ and $0.1 \mathrm{~N} \mathrm{HCl}(900 \mathrm{ml})$. Temperature is maintained at $37 \pm$ $0.5 \mathrm{C}$ and rotation speed of $50 \mathrm{rpm}$ is usually adjusted. Samples of drug dissolved are collected at pre-determined intervals and are analyzed by using UV-spectrophotometer. Despite its extensive use, dissolution test is still prone to noteworthy inaccuracy and tests let down [23, 24].

\section{CONCLUSION:}

The present review shows that oral fast disintegrating films are one of the novel approaches in the field of pharmaceutical sciences. They have improved acceptance and patient compliance with no risk of choking associated with better safety and efficacy in comparison with conventional dosage forms. The main idea behind formulation of ODFs was to cope with the difficulty in swallowing conventional oral dosage forms among pediatric, geriatric and psychiatric patients with dysphagia.Presently, ODFs are widely available for hypertension, acidity, allergy, pain, etc. reflecting their importance. Major advantages of such dosage form are their administration without the use of water fulfilling the need of target population seeking convenience in drug administration along with bypassing the hepatic metabolism, consequently, leading to improved therapeutic response.

\section{REFERENCES:}

1.Arya, A., Chandra, A., Sharma, V., Pathak, K., 2010. Fast dissolving oral films: an innovative drug delivery system and dosage form. Int. J. Chem. Tech. Res. 2, 576-583.

2. Bai, G., Armenante, P.M., Plank, R.V., Gentzler, M., Ford, K., Harmon, P., 2007. Hydrodynamic investigation of USP dissolution test apparatus II. J. Pharm. Sci. 96, 2327-2349.

3. Bala, R., Pawar, P., Khanna, S., Arora, S., 2013. Orally dissolving strips: A new approach to oral drug delivery system. Int. J. Pharm. Investig. 3, 67-73.

4. Chaudhary, H., Gauri, S., Rathee, P., Kumar, V., 2013. Development and optimization of fast dissolving oro-dispersible films of granisetron $\mathrm{HCl}$ using Box-Behnken statistical design. Bullet. Faculty Pharm. 51, 193-201.

5. Chauhan, I., Yasir, M., Nagar, P., 2012. Insights into polymers: film formers in mouth dissolving films. Drug Invent. Today 3, 56-73.

6.Desu, P.K., Brahmaiah, B., Nagalakshmi, A., Neelima, K., Nama, S., Baburao, C., 2013. An overview on rapid dissolving films. Asian J. Pharm. Res. 3, 15-23.
7. Dinge, A., Nagarsenker, M., 2008. Formulation and evaluation of fast dissolving films for delivery of triclosan to the oral cavity. AAPS Pharm. Sci. Tech. 9, 349-356.

8. Elmeshad1, A.N., Hagrasy, A.S.E., 2011. Characterization and optimization of orodispersible mosapride film formulation. AAPS Pharm. Sci. Tech. 12, 1384-1392.

9.Goel H, Rai P, Rana V, Tiwary A. Orally Disintegrating Systems: Innovations in Formulation and Technology. Recent Patents on Drug Delivery \& Formulation. 2008; 2(3):258-274.

10. Prabhu SC, Parsekar SD, Shetty A, Monteiro SS, Azharuddin M, Shabaraya AR. A Review on Fast Dissolving Sublingual Films for Systemic Drug Delivery. Int J Pharm Chem Sci. 2014; 3(2):50111.

11.Russo E, Selmin F, Baldassari S, Gennari C, Caviglioli G, Cilurzo F et al. A focus on mucoadhesive polymers and their application in buccal dosage forms. Journal of Drug Delivery Science and Technology. 2015;32:113-125.

12. Wening $\mathrm{K}$ Breitkreutz J. Oral drug delivery in personalized medicine: Unmet needs and novel approaches. Int J Pharm. 2011;404(1-2):1-9.

13. Hariharan M, Bogue A. Orally dissolving film strips (ODFS): the final evolution of orally dissolving dosage forms. Drug Deliv Technol. 2009 Feb;9(2):24-9.

14. Sakellariou P, Rowe R, White E. An evaluation of the interaction and plasticizing efficiency of the polyethylene glycols in ethyl cellulose and hydroxypropyl methylcellulose films using the torsional braid pendulum. IntJ Pharm. 1986; 31(1-2):55-64.

15. Patil PC, Shrivastava SK, Vaidehi S, Ashwini P. Oral Fast Dissolving drug delivery system: A modern approach for patient compliance. Int J Drug Regulatory Affairs. 2014 Jun 1; 2(2):4960.

16. Rekha MR, Sharma CP. Pullulan as a promising biomaterial for biomedical applications: a perspective. Trends BiomaterArtif Organs. 2007; 20(2):116-21.

17. U.S. Congress, Office of Technology Assessment, Biopolymers: Making Materials Nature's Way-Background Paper, OTA-BP-E102 (Washington, DC: U.S. Government Printing Office, 1993).

18. Saini S, Rana AC, Gupta S. Optimization of formulation of fast dissolving films made ofpullulan polymer. Int J Pharm Sci Rev Res. 2011;9(1):127-31.

19.laudia, A. R. B., Bello-Perez, L. A.Gacia, M. A.; Martino, M. N.; Solorza-Feria, J.; Zaritzky, N. E. Carbohyd. Polym. 2005; 60: 235244.

20. Laohakunjit N, Noomhorm A. Effect of plasticizers on mechanical and barrier properties of rice starch film. Starch/Staerke 2004; 56:348-356.

21. Wu Y, Weller C, Hamouz F, Cuppett S, Schnepf M. Moisture Loss and Lipid Oxidation for Precooked Ground-Beef Patties Packaged in Edible Starch-Alginate-Based Composite Films. Journal of Food Science. 2001;66(3):486-493.

22. El-SetouhyDEl-Malak N. Formulation of a Novel Tianeptine Sodium Orodispersible Film. AAPS PharmSciTech. 2010; 11(3):1018-1025.

23. Kunte S, Tandale P. Fast dissolving strips: A novel approach for the delivery of verapamil. J Pharm Bio Sci. 2010;2(4):325-328.

24. Ramani C.C., Puranik P.K., Dorl A.K. Study of diabetic acid as matrix forming material. Int J Pharm. 1996; 137:11-19. 